\title{
Single-machine Integrated Production Preventive Maintenance Scheduling: A Simheuristic Approach
}

\author{
${ }^{1}$ Nurul Nadiah Abdul Halim*, ${ }^{2}$ S. Sarifah Radiah Shariff and ${ }^{3}$ Siti Meriam Zahari \\ ${ }^{1,2,3}$ Centre for Statistics and Decision Science Studies \\ Faculty of Computer and Mathematical Sciences \\ ${ }^{2}$ Malaysia Institute of Transport (MITRANS) \\ Universiti Teknologi MARA \\ 40450, Shah Alam, Selangor, Malaysia \\ ${ }^{*}$ Corresponding author: nadiahalim_87@yahoo.com
}

Article history

Received: 14 March 2019

Received in revised form: 21 July 2020

Accepted: 23 July 2020

Published online: 1 August 2020

\begin{abstract}
Preventive maintenance (PM) planning becomes a crucial issue in the real world of the manufacturing process. It is important in the manufacturing industry to maintain the optimum level of production and minimize its investments. Thus, this paper focuses on multiple jobs with a single production line by considering random machine breakdown time. The aim of this paper is to propose a good integration of production and PM schedule that will minimize total completion time. In this study, a hybrid method, which is a genetic algorithm (GA), is used with the Monte Carlo simulation (MCS) technique to deal with the uncertain behavior of machine breakdown time. A deterministic model is adopted and tested under different levels of complexity. Its performance is evaluated based on the value of average completion time. The result clearly shows that the proposed integrated production with PM schedule can reduce the average completion time by $11.68 \%$ compared to the production scheduling with machine breakdown time.
\end{abstract}

Keywords Machine Failure; Multiple-production; Preventive Maintenance; Single Line; Simheuristic.

Mathematics Subject Classification 90B35

\section{Introduction}

Scheduling can be defined as the process of assigning limited resources to a set of jobs need to be accomplished. In production systems, jobs represent the tasks, which need to be processed on machines, which represent the resources, [1]. It plays an important role in a wide range of industries, for instance, the oil industry, the pharmaceutical and manufacturing sectors as well as in the transportation sectors. Production scheduling is the core process of every manufacturing organization. Nowadays, it becomes the most crucial issues in managing production scheduling properly to remain competitive in the industry. Thus, 
the manufacturing companies need to stay competitive in a highly dynamic and changing manufacturing environment and maintain profitable in the market by continuously converting raw materials into value-added products that meet the customers' needs.

The most difficult problem in this area is to sequence the jobs. In scheduling, each job is formed by a sequence of consecutive operations, each operation requires exactly one machine, machines are continuously available and can process one operation at a time without interruption.

Improper planning and scheduling the production results in higher operating costs. Moreover, optimal scheduling can help the company in allocating the products to machines, the sequencing of processing products on each machine, and the production quantity of each product in such a way that they can optimize the production schedule to achieve their goals. Thus, proper production scheduling cannot be denied.

For the manufacturing sector, it will face uncertain scheduling problems for example machine breakdown due to incorrect PM scheduling. This will lead to inefficient production process and unable to meet customers' demand because the production system is not able to run smoothly. Maintenance process needs to be done when the machine breakdown. In any manufacturing company, the machine breakdown means cost to the company [2]. Hence, it is important to prevent the machine breakdown. As production scheduling becomes one of the most critical issues in the planning and managing of manufacturing processes, various types of production scheduling models have been studied to improve scheduling production. Metaheuristic methods such as, GA [3,4,5], Simulated Annealing (SA) [6], Scatter Search (SS) [7] were used to solve the problem. Similarly, various simulation methods $[8,9,10]$ were also widely used to solve relevant manufacturing problems. The existing methods have shown several benefits such as cost reductions, increased utilization, and many more. Despite of various benefits, there are some drawbacks; for example, in SA, its searching-time is very slow and not suitable for complex optimization problem while SS updates the population using deterministic rules. Due to the complexity of this system, it is suggested to explore the hybridization of metaheuristic methods that will produce a good schedule in a reasonable time. In recent years, the hybrid methods between metaheuristics itself have led to improved results than classical dispatching [11]. However, the metaheuristics method assumes that the input is deterministic in nature [12]. Thus, an alternative hybrid method based on simulation and metaheuristic techniques (known as simheuristics technique) should be considered to cater for the uncertainty in the real-world problem [13]. A simheuristics method based on Monte Carlo simulation (MCS) and metaheuristic techniques for production scheduling models had been studied to improve scheduling production and represent the real-world problems [14,15].

There are some constraints that occur in production scheduling of a company; such as the inability to meet job due dates for machine scheduling that will affect the daily production, inability to cope with variations in demand, and also machines maintenance and breakdown. A proper production and PM scheduling are important to cope with these problems.

Thus, the main objective of this paper is to propose a good integration of production and PM schedule that will minimize the total completion time. This paper focuses on multiple jobs with a single machine by considering stochastic machine breakdown time [5,13]. In this type of problem, it can be divided into two phases in which the first part is to predict the machine breakdown (stochastic in nature, solve using simulation) and the second part is to schedule the production on multiple jobs which can be very complex (solve using metaheuristic). A 
simheuristics algorithm based on MCS and GA is implemented in this study for solving this stochastic optimization problem. GA is used in this study because it is a simple algorithm which able to generate a good solution that can achieve near optimal solution. In addition, the used of MCS technique is to deal with the stochastic behavior of machine breakdown time.

\section{Research Methodology}

This section explains the steps involved in this study. This study used the basic model developed by [16] in determining the significant result that helps the top management of the relevant organization in developing a proper job sequence. The simheuristics approach by [17] is adopted in this study. The study relies on GA and MCS techniques. In this study, GA is implemented to explore the combinatorial optimization problems, whereas MCS technique is used to enable the assessment of solutions in a dynamic environment. In this study, the priority of jobs was chosen based on the minimum average of completion time because it shows the ability of the company to serve the customers within the time span given.

Data used in this study is taken from the literature, [16] and summarized in Table 1. There are six different jobs that are needed to be scheduled in a single line production and suppose that preempting one job for another is not permitted. It clearly shows that Job 2 has the shortest processing time ( 7 hours) and with longest processing time is Job 3 (42 hours).

Table 1: The Original Data (Hours)

\begin{tabular}{|l|c|c|c|c|c|c|}
\hline Job & Job 1 & Job 2 & Job 3 & Job 4 & Job 5 & Job 6 \\
\hline $\begin{array}{l}\text { Processing time for six different jobs, } \\
\mathrm{P}_{j} \text { (hours) }\end{array}$ & 25 & 7 & 42 & 36 & 18 & 29 \\
\hline
\end{tabular}

\section{$2.1 \quad$ Study Settings}

This section describes the research design of this study. There are three phases in this study:

i Phase 1: The individual production scheduling problem.

ii Phase 2: Simulation study for machine breakdown and machine's repair time.

iii Phase 3: The proposed integrated production and PM scheduling approach.

\subsubsection{Phase 1: The Individual Production-scheduling Problem}

In Phase 1, GA is used to obtain the best initial schedule without machine breakdown. GA was first introduced by Holland [18]. GA technique is inspired by natural evolution such as selection, crossover and mutation to generate optimal solution. It becomes the most successful method to solve most optimization problems [19]. In this study, the framework of GA from $[5,20]$ is adopted as the solution searching process. Figure 1 shows the adopted framework of GA. 


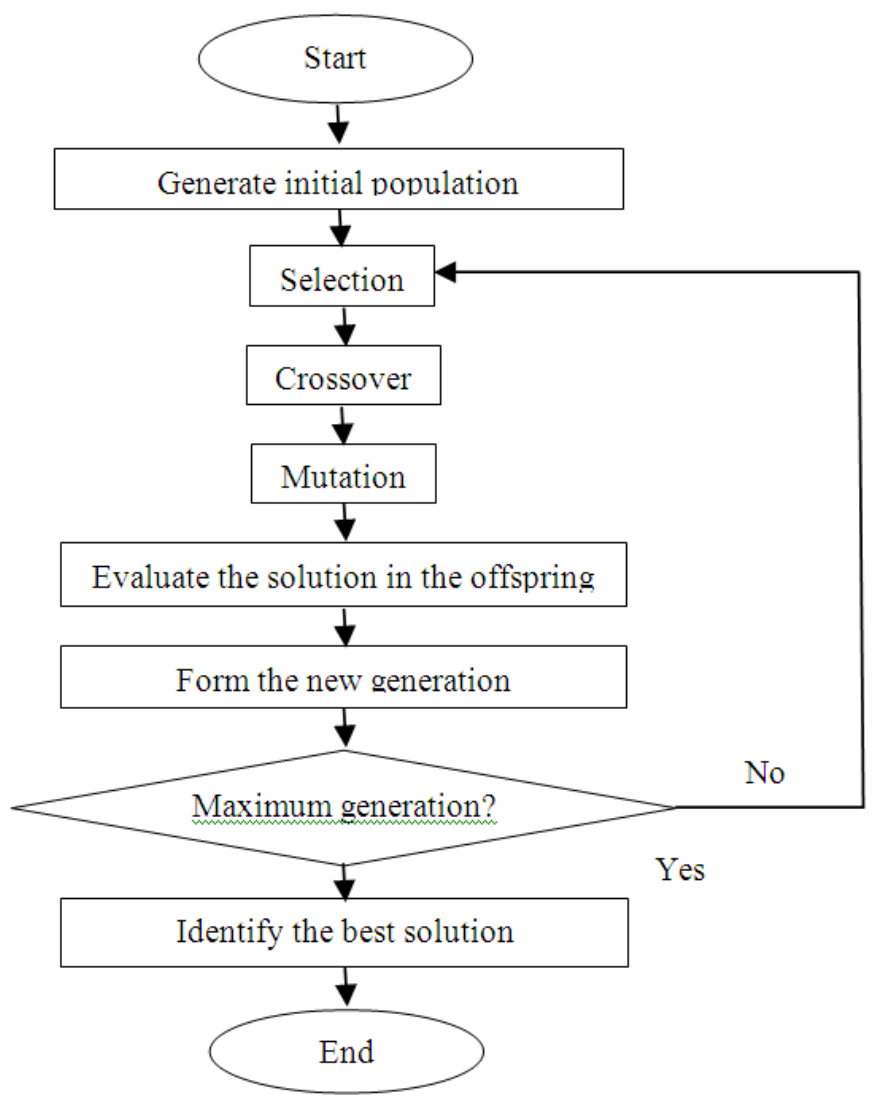

Figure 1: Adopted Framework of GA

\section{Step 1: Initialization Stage (Chromosomal Representation)}

The sequence of the job is represented as a chromosome, which is a string consisting of $\mathrm{N}$ integer numbers. The numbers indicate the number of jobs in the sequence. In this case, one to six represent Job 1 to Job 6 respectively. Figure 2 shows an example of the job sequence, represented as a chromosome. In this example, the job sequence starts with Job 4, followed by Job 3, Job 2, Job 5, Job 6 and Job 1.

\begin{tabular}{|l|l|l|l|l|l|}
\hline 4 & 3 & 2 & 5 & 6 & 1 \\
\hline
\end{tabular}

Figure 2: A Chromosome Example

\section{Step 2: Initial Population}

An initial population, $N_{p}$ is randomly generated. In this study, $N_{p}$ refers to the number of job sequence randomly generated. 


\section{Step 3: Objective Function}

The objective function is to minimize the average expected total completion time. By using the following notations:

$$
\begin{aligned}
x_{i j} & =\left\{\begin{array}{l}
1, \text { if the } i^{\text {th }} \text { job performed is job } j \\
0, \text { otherwise }
\end{array}\right. \\
i & =1,2, \ldots, n \text { (job sequence) } \\
j & =1,2, \ldots, n \text { (number of jobs) } \\
C_{i} & =\text { completion time at the } i^{t h} \text { job } \\
p_{i} & =\text { processing time at the } i^{t h} \text { job } \\
p_{j} & =\text { processing time of job } j
\end{aligned}
$$

The objective function can be written as in Equation (1)

$$
\text { Minimize } E\left(C_{i}\right)=\sum_{i=1}^{n}\left(p_{i}+C_{i-1}\right)
$$

where $p_{i}=\sum_{j=1}^{n} p_{j} x_{i j}$

\section{Step 4: Genetic Operations Implementation Process}

The section explains the genetic implementation process in this study. The genetic operations process includes the selection of chromosomes, crossover operator and followed by mutation process.

i. Selection In this study, roulette wheel selection is implemented select a number of chromosomes for crossover operation.

\section{ii. Crossover}

Two-point crossover is used in this study. First, two cut points are determined randomly from the parent chromosomes. The genes in between the two cut points are swapped together between the parent chromosomes to generate a new child. Crossover operator has a probability rate or known as the probability of crossover $(P c)$. $P c$ gives the possibility of applying a crossover to produce an offspring from a number of selected parents. Figure 3 shows an example of a two-point crossover.

However, both offspring are infeasible since Job 5 in Offspring 1 and Job 1 in Offspring 2 is missing. Therefore, an offspring repair procedure is conducted to both offspring by replacing the repeated genes with the missing one based on the order they appear in the other parent as shown in Figure 4.

\section{iii. Mutation}

Mutation process is important to keep diversity in a population. This study implements a simple inversion mutation. In inversion mutation, two points are randomly selected from an individual. Then, the jobs in that segment are reinserted in the reverse order as shown in Figure 5. 


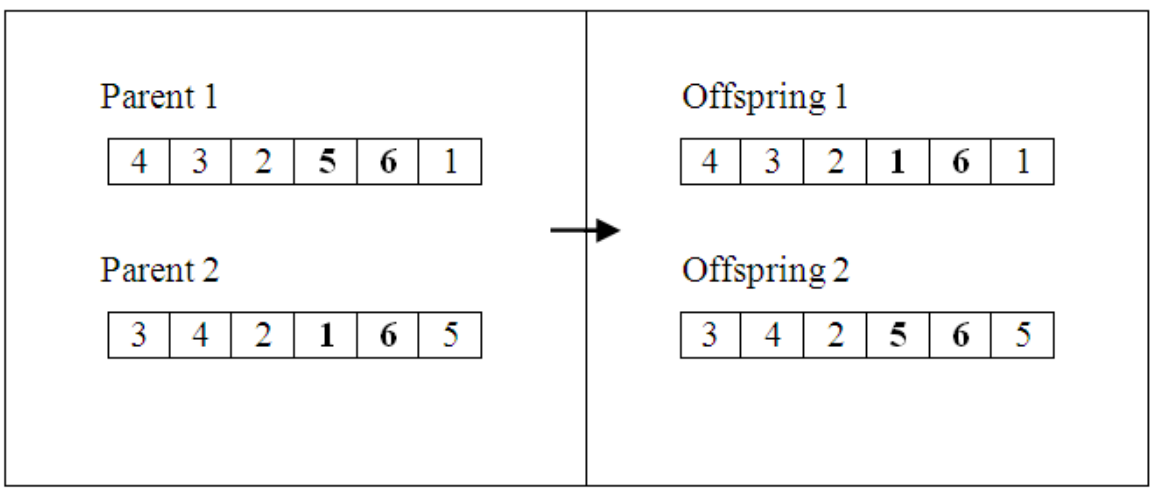

Figure 3: Example to Illustrate Two-point Crossover

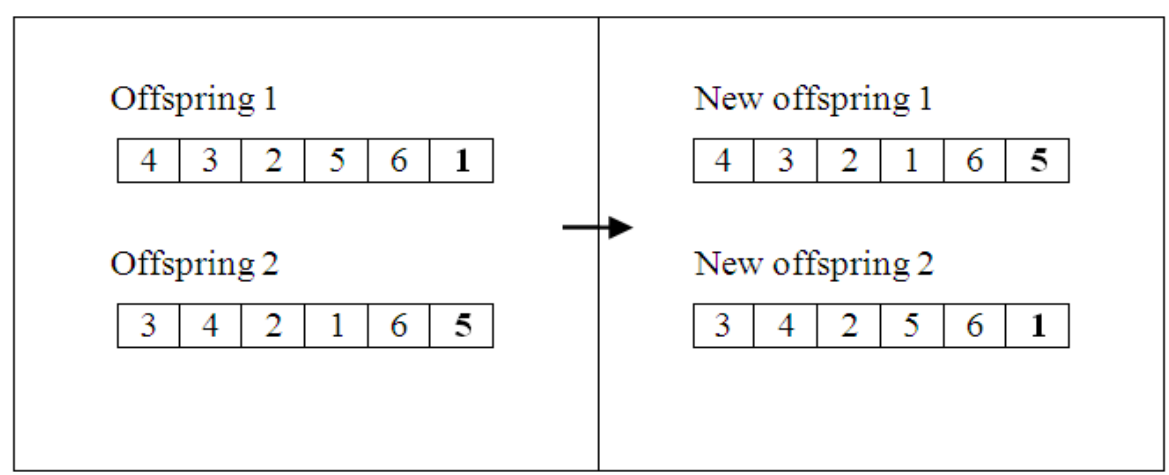

Figure 4: Example to Illustrate Two-point Crossover after Repairing Procedure

\section{Step 5: Control Parameters}

The most important step in GA is control parameters. It is important to identify the number of individuals in the population (population size), and the rate of crossover and mutation carefully. A large population size leads to the best probability of convergence to a global optimal solution compared to small population size. However, a large number of populations leads to higher computation time. A lower crossover rate can reduce the speed of convergence to the global optimal solution area. On the other hand, a high value of mutation rate can cause instability and too low of mutation rate leads to difficult for a GA to find a global optimal solution. Thus, the population size used in this study equals 100, the crossover probability, $P c=0.8$, the mutation rate, $P m=0.1$. The algorithm is terminated after the number of generations reaches 720 times. The number of generations is determined based on the number of possible job sequences $n$ ! where $n$ is the number of jobs [15].

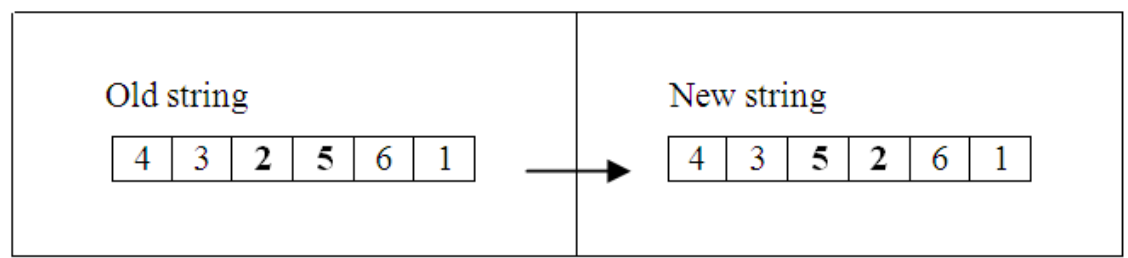

Figure 5: Inversion Mutation Example 


\section{Step 6: Identify the Best Solution}

The best 10 job sequences are chosen based on the lowest average completion time. The GA approach is implemented using $\mathrm{R}$ software.

\subsubsection{Simulations Study for Machine Breakdown Time}

In this study, MCS is used to address the machine breakdown time and to estimate the machine repair time. There are five steps in MCS [13].

i. Understand the problem under study and data collection.

ii. Set-up probability distribution for machine breakdown time.

iii. Find the cumulative probability distribution for machine breakdown time.

iv. Generate random number to represent the real situation of the problem under study.

v. Simulate the real process using the random number obtained.

The simulation process is conducted using Microsoft Excel. The machine breakdown time follow continuous probability as discussed below.

\subsubsection{The Estimation Time for Machine Breakdown and Machine Repair Time}

According to [21], the elapsed time for machine breakdown follows continuous probability. In this case, the machine's failure rate is defined by the following Weibull distribution $[2,16,22,23]$. By using the following notations:

$$
\begin{aligned}
& \mathrm{F}(\mathrm{t})=\text { the expected number of machine failure in one operating cycle } \\
& \begin{aligned}
\theta & =\text { scale parameter } \\
\beta & =\text { shape parameter } \\
\lambda(t) & =\text { system's failure rate at time } \\
t \quad & =\text { time of machine breakdown }
\end{aligned}
\end{aligned}
$$

The expected number of machine failure in one operating cycle can be written as in Equation (2)

$$
F(t)=\int_{0}^{t} \lambda(t) d t,
$$

where

$$
\lambda(t)=\frac{\beta}{\theta} \cdot\left(\frac{t}{\theta}\right)^{\beta-1} .
$$

The model above is solved to find the solution for production scheduling with machine breakdowns. The random numbers in this problem are generated using the RAND () function in Excel. Maintenance process is done when the machine breakdowns. The time for maintenance activity is fixed to 15 hours.

\subsubsection{Phase 3: The Integrated Production and PM Scheduling}

This section explains the proposed integrated production and PM scheduling. Initially, the job sequence is obtained based on the individual production scheduling. The detailed process of 
individual production scheduling was briefly explained in Section 2.1.1. Then, PM activities are scheduled and the optimal time intervals for maintenance activities are determined. The estimation PM time interval and time for performing PM is discussed in the following section.

\subsubsection{Estimation PM Time Interval}

The time interval for PM is estimated based on [16]. By using the following notations: The time interval for PM can be expressed as in Equation (3)

$$
t^{*}=\theta \cdot\left[\frac{t_{p m}}{t_{m r} \cdot(\beta-1)}\right]^{(1 / \beta)}
$$

Based on $[16,22]$ assumed the value for $\theta=100, \beta=2, t_{p m}=5$ and $t_{m r}=15$. Thus, the estimated time interval for PM activity is 57.77. It means that for every 57.77 hours of the machine's operation, one PM activity should be conducted.

\subsubsection{Time for Performing Preventive Maintenance Estimation}

According to [16] the time for performing PM activity prior to $i^{\text {th }}$ job can be calculated as in Equation (4), where $a$ is the effective age of the machine:

$$
t_{p, a}=5 \times((a+500) / 500)^{3}
$$

\subsection{Computational Experiment}

The efficiency of the proposed integrated production with PM scheduling is compared to the production scheduling model with machine breakdown without PM scheduling. The methodology presented has been implemented in Microsoft Excel.

\section{Case 1: An Individual Production Scheduling without Preventive Maintenance}

For the individual production scheduling problem, the machine is assumed to be always available for production during time horizon and hence, the PM planning is neglected in this case. The initial production schedule without machine breakdown and PM is obtained using GA method as described in Section 2.1.1. The best 10 jobs sequence is chosen based on the lowest average of completion time.

Case 2: Production Scheduling with Machine Breakdown and Machine Repair Time

Particularly, in this case, the production scheduling problem is based on the unavailability time for the machine to execute the process due to machine breakdown that will interrupt the daily production. The job sequences are rescheduled by considering the machine breakdown. Machine breakdown are uncertain and can occur at any time during the production schedule. Thus, a simulation based on the MCS technique (refer Section 2.1.2) is done to address the machine breakdown time. The maintenance process is done when the machine breakdown.

\section{Case 3: The Proposed Integrated Production with PM Scheduling}

This case discusses the influenced of PM schedule in improvement of individual production scheduling model. First, the job sequence is obtained based on the individual production 
scheduling without considering machine breakdown and PM schedule. The PM schedule is solved using Microsoft Excel. The estimation PM time interval and time for performing PM has been discussed in detail in Section 2.1.3.

\section{Results and Discussion}

This section discusses the results and the analysis of scheduling. As mentioned earlier, there are three cases analyzed in this study which includes individual production without maintenance scheduling, production scheduling model with machine breakdown and integrated production and PM scheduling. The methodology presented has been implemented in R software and Microsoft Excel. The computation test is carried out on an Intel (R) Core (TM) i5 - 5200 CPU @ $2.20 \mathrm{GHz}, 2.19 \mathrm{GHz}$ and $8 \mathrm{~GB}$ RAM operating under Windows 10 Pro.

\section{Case 1: Production Scheduling without Preventive Maintenance}

Table 2 shows a comparison of the average completion time for the best 10 job sequences. The best jobs sequence is chosen based on the lowest average of completion time. The lowest of the average completion time is 72.17 hours. Thus, the best jobs sequence obtained starts with Job 2, followed by Job 5, Job 1, Job 6, Job 4 and Job 3.

Table 2: The Best 10 Job Sequences and the Average Completion Time

\begin{tabular}{|c|c|c|}
\hline No & Job Sequence & Average Completion Time (hours) \\
\hline 1 & $2-5-1-6-4-3$ & $\mathbf{7 2 . 1 7}$ \\
\hline 2 & $2-5-6-1-4-3$ & 72.83 \\
\hline 3 & $2-5-1-6-3-4$ & 73.17 \\
\hline 4 & $2-1-5-6-4-3$ & 73.33 \\
\hline 5 & $2-5-1-4-6-3$ & 73.33 \\
\hline 6 & $2-5-6-1-3-4$ & 73.83 \\
\hline 7 & $5-2-1-6-4-3$ & 74.00 \\
\hline 8 & $2-1-5-6-3-4$ & 74.33 \\
\hline 9 & $2-1-5-4-6-3$ & 74.50 \\
\hline 10 & $2-5-6-4-1-3$ & 74.67 \\
\hline
\end{tabular}

\section{Case 2: Production Scheduling with Machine Breakdown and Machine's Repair} Time

The steps involved in production scheduling with machine breakdown and machine's repair time has been discussed in detail in Section 2.1.2. The random number intervals for machine breakdown is shown in Table 3. Table 4 shows the outcome of the production scheduling with machine breakdown time using MCS. The average completion time for production with machine breakdown and the machine's repair time also shown in Table 4. From Table 4, it shows job sequence start from Job 2, Job 5, Job 1, Job 6, followed by Job 4 and the last one is Job 3. The job sequence in Case 2 is the same as job 
sequence generated in Case 1. Based on the equation (2) in Section 2.1.2.1, the expected number of machine failure in one operating cycle in this study is three times. This study assumed that the probability of machines breakdown is equally likely to the machine without breakdown. Based on the MCS, the machine breakdown occurs during Job 5, Job 4 and Job 3. The machine's repair time is fixed to 15 hours. From the MCS result it shows that the average completion time is 92.17 hours.

Table 3: Random Number Intervals for Machine Breakdown

\begin{tabular}{|c|c|c|c|}
\hline $\begin{array}{c}\text { Machine's } \\
\text { condition }\end{array}$ & $\begin{array}{c}\text { Probability } \\
\text { distribution }\end{array}$ & $\begin{array}{c}\text { Cumulative probability } \\
\text { distribution }\end{array}$ & $\begin{array}{c}\text { Random number } \\
\text { interval }\end{array}$ \\
\hline Without Breakdown & 0.50 & 0.50 & $00-50$ \\
\hline Breakdown & 0.50 & 1.00 & $51-99$ \\
\hline
\end{tabular}

Table 4: Production Scheduling with Machine Breakdown, Machine's Repair Time and Average Completion Time

\begin{tabular}{|c|c|c|c|c|c|}
\hline Job & $\begin{array}{c}\text { Random } \\
\text { Number }\end{array}$ & $\begin{array}{c}\text { Machine } \\
\text { Breakdown? } \\
\text { Yes/No) }\end{array}$ & $\begin{array}{c}\text { Processing } j \\
\text { Time of Job } j\end{array}$ & $\begin{array}{c}\text { Machine's } \\
\text { Repair Time } \\
\text { (hours })\end{array}$ & $\begin{array}{c}\text { Completion } \\
\text { Time of Job } j \\
\text { (hours) }\end{array}$ \\
\hline 2 & 0.46 & No & 7.00 & 0.00 & 7.00 \\
\hline 5 & 0.80 & Yes & 18.00 & 15.00 & 40.00 \\
\hline 1 & 0.25 & No & 25.00 & 0.00 & 65.00 \\
\hline 6 & 0.17 & No & 29.00 & 0.00 & 94.00 \\
\hline 4 & 0.90 & Yes & 36.00 & 15.00 & 145.00 \\
\hline 3 & 0.75 & Yes & 42.00 & 15.00 & 202.00 \\
\hline Average & & & & & 92.17 \\
\hline
\end{tabular}

\section{Case 3: Integrated Production with Preventive Maintenance Scheduling}

Table 5 shows the job sequence being rescheduled by inserting PM activities. The optimal time interval for PM activity is 57.77 as calculated in Section 2.1.3.1. It means that PM activity should be done every 57.77 hours of the machine's operation. PM can restore the machine to be as good as new condition. It means that the machine is renewed, and its effective age returns to zero. Based on PM activity interval, one maintenance activity is conducted before the first job (Job 2). The machine's initial effective age, $a_{o}$ for a single machine is 88 [16] and the time for PM activity is calculated as described in Equation (4), Section 2.1.3.2. The first PM activity is 8 hours. The next PM activity should be performed after Job 5 (PM time $=6$ hours), Job 6 (PM time $=7$ hours $)$ and Job 4 (PM time $=6$ hours). The average completion time for the integrated production and PM schedule is 81.40 hours which is smaller than the production scheduling with machine breakdown. 
Table 5: Job Sequence Integrated with Preventive Maintenance Planning and the Average Completion

\begin{tabular}{|c|c|c|}
\hline Job & Processing Time (hours) & Completion Time (hours) \\
\hline PM & 8.00 & 8.00 \\
\hline 2 & 7.00 & 15.00 \\
\hline 5 & 18.00 & 33.00 \\
\hline PM & 6.00 & 39.00 \\
\hline 1 & 25.00 & 64.00 \\
\hline 6 & 29.00 & 93.00 \\
\hline PM & 7.00 & 100.00 \\
\hline 4 & 36.00 & 136.00 \\
\hline PM & 6.00 & 142.00 \\
\hline 3 & 42.00 & 184.00 \\
\hline \multicolumn{2}{|c|}{ Average } & 81.40 \\
\hline
\end{tabular}

\subsection{Summary of Results}

Table 6 shows the comparison of the average completion time between production scheduling with machine breakdown and the machine's repair time, and integrated production with PM scheduling. It clearly shows that the proposed integrated production with PM scheduling shows a lower average completion time ( 81.40 hours) compared to production scheduling with machine breakdown (92.17 hours) and has reduced the average completion time by $11.68 \%$. The result of this study proves that the integrated production with PM scheduling can reduce the average completion time as mentioned in $[16,22]$. It shows that the integrated production with PM scheduling is better than the production scheduling without PM.

Table 6: Summary of Results

\begin{tabular}{|l|c|}
\hline Types of Scheduling & Average Completion Time of Job $j$ (hours) \\
\hline $\begin{array}{l}\text { Production scheduling with machine } \\
\text { breakdown and machine's repair time }\end{array}$ & 92.17 \\
\hline $\begin{array}{l}\text { Integrated production with PM } \\
\text { scheduling }\end{array}$ & 81.40 \\
\hline $\begin{array}{l}\text { Percentage reduction in average total } \\
\text { completion time }\end{array}$ & $11.68 \%$ \\
\hline
\end{tabular}




\section{Conclusion}

The main objective of this study is to schedule the multiple productions with a single line in such a way that the total average completion time can be minimized. The integrated procedures that combine MCS and GA are developed to deal with random machine breakdown time in this study. The computational experiments carried out in this paper show how to schedule the production properly according to various factors such as the various level in the random machine breakdown times. This study shows that the average total completion time can be reduced by $11.68 \%$.

As this study focuses on a single machine scheduling problem, it can be extended to a multiple machines problem or consider other uncertain situation likes different machine's setup times, and variation in machine's repair time.

\section{Acknowledgements}

This work has been fully supported by the Ministry of Education Malaysia and GE STEM grant with vot no. 07397 .

\section{References}

[1] Silva, J. D. L., Burke, E. K. and Petrovic, S. An Introduction to Multiobjective Metaheuristics for Scheduling and Timetabling. Berlin: Springer. 2004.

[2] Yulan, J., Zuhua, J. and Wenrui, H. Multi-objective integrated optimization research on preventive maintenance planning and production scheduling for a single machine. International Journal Advanced Manufacturing Technology. 2008. 39: 954-964.

[3] Fattahi, P. and Fallahi. Dynamic scheduling in flexible job shop systems by considering simultaneously efficiency and stability. CIRP Journal of Manufacturing Science and Technology. 2010. 2: 114-123.

[4] Lee, S. and Ni, J. Genetic algorithm for job scheduling with maintenance consideration in semiconductor manufacturing process. Mathematical Problems in Engineering. 2012. Article ID 875641, doi:10.1155/2012/875641.

[5] Lu, Z., Cui, W. and Han, X. Integrated production and preventive maintenance scheduling for a single machine with failure uncertainty. Computers and Industrial Engineering. 2015. 80: 236-244.

[6] Sadagheih, A. Scheduling problem using a genetic algorithm, simulated annealing and the effects of parameter values on GA performance. Applied Mathematical Modelling. 2006. 30: $147-154$.

[7] Jaradat, G. M., Ayob, M. and Ahmad, Z. Scatter search metaheuristics for postenrolment course timetabling problems: A Review. International Journal of Advancements in Computing Technology (IJACT). 2013. 5: 118-125.

[8] Parthanadee, P. and Buddhakulsomsiri, J. Simulation modeling and analysis for production scheduling using real-time dispatching rules: A case study in canned fruit industry. Computers and Electronics in Agriculture. 2010. 70: 245-255. 
[9] Varela, M. L. R., Trojanowska, J., Caro-Silva, S., Costa, N. M. L. and Machado, J. Comparative simulation study of production scheduling in the hybrid and parallel flow. Management and Production Engineering Review. 2017. 8: 69- 80.

[10] Maram, V., Nawawi, M. K. M., Rahman, S. A. and Sultan, S. J. Simulation as planning tool for job-shop production environment. 2015. In AIP Conference Proceedings 1691. 030018. doi: 10.1063/1.4937037.

[11] Joon-Yung, M., Kitae, S. and Jinwoo, P. Optimization of production scheduling with time-dependent and machine-dependent electricity cost for industrial energy efficiency. International Journal Advanced Manufacturing Technology. 2013. 68: 523-535.

[12] Chica, M., Juana, A. A., Cord'on, O. and Kelton, W. D. Why Simheuristics? Benefits, Limitations, and Best Practices when Combining Metaheuristics with Simulation. 2015. Retrieved from http://openaccess.uoc.edu/webapps/o2/bitstream/10609/85185/1/SSRNid2919208.pdf.

[13] Calvet, L., Fernandez-Viagas, V., Framinan, J. M. and Juan, A. A. Combining simulation with metaheuristics in distributed scheduling problems with stochastic processing time. In Proceeding of the Winter Simulation Conference. 2016. 2347-2357.

[14] Juan, A. A., Barrios, B. B., Vallada, E., Riera, D. and Jorba, J. Simheuristic algorithm for solving the permutation flow shop problem with stochastic processing times. Simulation Modelling Practice and Theory. 2014. 46: 101-117.

[15] Gonzalez-Neira, E. M., Ferone, D., Hatami, S. and Juan, A. A. A biased-randomized simheuristic for the distributed assembly permutation flowshop problem with stochastic processing times. Simulation Modelling Practice and Theory. 2017. 79: 23-36.

[16] Pan, E., Liao, W. and Xi, L. Single-machine-based production scheduling model integrated preventive maintenance planning. International Journal Advanced Manufacturing Technology. 2010. 50: 365-375.

[17] Juan A. A., Faulin J, Grasman SE, Rabe M, Figueira, G. A review of simheuristics: Extending metaheuristics to deal with stochastic combinatorial optimization problems. Operations Research Perspectives. 2015. 2: 62-72.

[18] Holland, J. H. Adaptation in Natural and Artificial Systems. MI. Ann Arbor: University of Michigan Press. 1975.

[19] Rashid, H., Siam, F. M., Maan, N. and Rahman, W. N. W. A. Parameter estimation for a model of ionizing radiation effects on targeted cells using genetic algorithm and pattern search method. Malaysian Journal of Industrial and Applied Mathematics. Special Issue. 2018. 1-13.

[20] Nidhiry, N. M. and Saravanan, R. Evaluation of genetic algorithm approach for optimization of flexible manufacturing system. International Journal of Engineering Research and Application (IJERA). 2012. 2: 437-446.

[21] Taylor, B. W. Introduction to Management Science. 10th Edition. Prentice Hall: Pearson Education, Inc. 2010.

[22] Cassady, C. R. and Kutanoglu, E. Integrating preventive maintenance planning and production scheduling for a single machine. IEEE Transactions on Reliability. 2005. 54: 304-309. 
[23] Ye, J. and Ma, H. Multiobjective joint optimization of production scheduling and maintenance planning in the flexible job-shop problem. Mathematical Problems in Engineering. 2015. 1-9. 